Supplement of Biogeosciences Discuss., 12, 16255-16283, 2015

http://www.biogeosciences-discuss.net/12/16255/2015/

doi:10.5194/bgd-12-16255-2015-supplement

(C) Author(s) 2015. CC Attribution 3.0 License.

(c) (1)

Supplement of

\title{
Composition of ammonia-oxidizing archaea and their contribution to nitrification in a high-temperature hot spring
}

\section{S. Chen et al.}

Correspondence to: X. Peng (xtpeng@ sidsse.ac.cn)

The copyright of individual parts of the supplement might differ from the CC-BY 3.0 licence. 


\section{Supplement}

\subsection{Bacterial community composition and phylogenetic analysis.}

Compared to the archaeal representatives of this hot spring environment, the bacterial 16S rRNA gene composition and the abundance of OTUs showed a higher diversity in community composition. All of the bacterial sequences could be classified into several momophyletic groups: Aquificae, Acidobacteria, Chlorobi, Chloroflexi, Cyanobacteria, Candidate division Op1, Deinococcus-Thermus, Firmicutes, Nitrospirae, and Proteobacteria (alpha and beta).

Both clone libraries were dominated by Chloroflexi and Firmicutes: these two groups composed 22 to 33\% of the total bacterial 16S rRNA gene sequences (Fig 3). Firmicutes, which was found in various environments, such as grassland soil, seafloor sediments, and hot springs (Kasahara and Hattori, 1991; Gontang, et al., 2007), includes taxa that are related to Bacillus sp and Paenibacillus sp. In the Chloroflexi group, 31 clone sequences from SS and BS (23 and 8, respectively) were related to sequences retrieved from hot spring environments (Hanada, et al., 2002). In addition, Acidobacteria was another dominant phylum in samples collected from the bottom sediment. Thirty-four sequences from BS-B20 were related to bacterium clone A3DB-A9 (DQ645247), which was obtained from a highly arsenic, circumneutral hot water spring of the Alvord Desert (GenBank description). However, Nitrospirae and cyanobacteria were observed only in the upper side sediments library; the cyanobacterial forms were closely related to filamentous, thermophilic cyanobacterium. The sequences of Nitrospirae were closely affiliated with Thermodesulfovibrio hydrogeniphilus. It was documented that this strain was a new, thermophilic, sulfate-reducing bacterium isolated from a Tunisian hot spring (Haouari, et al., 2008).

Approximately $15 \%$ of the total bacterial clone sequences were affiliated with minor groups, such as Aquificae, Deinococcus-Thermus, Chlorobi, Proteobacteria, and 
Candidate division Op1. Most of the sequences were related to clones retrieved from thermal environments in China, the USA, and other continental hot springs (Kanokratana, et al., 2004; Peng et al., 2013).

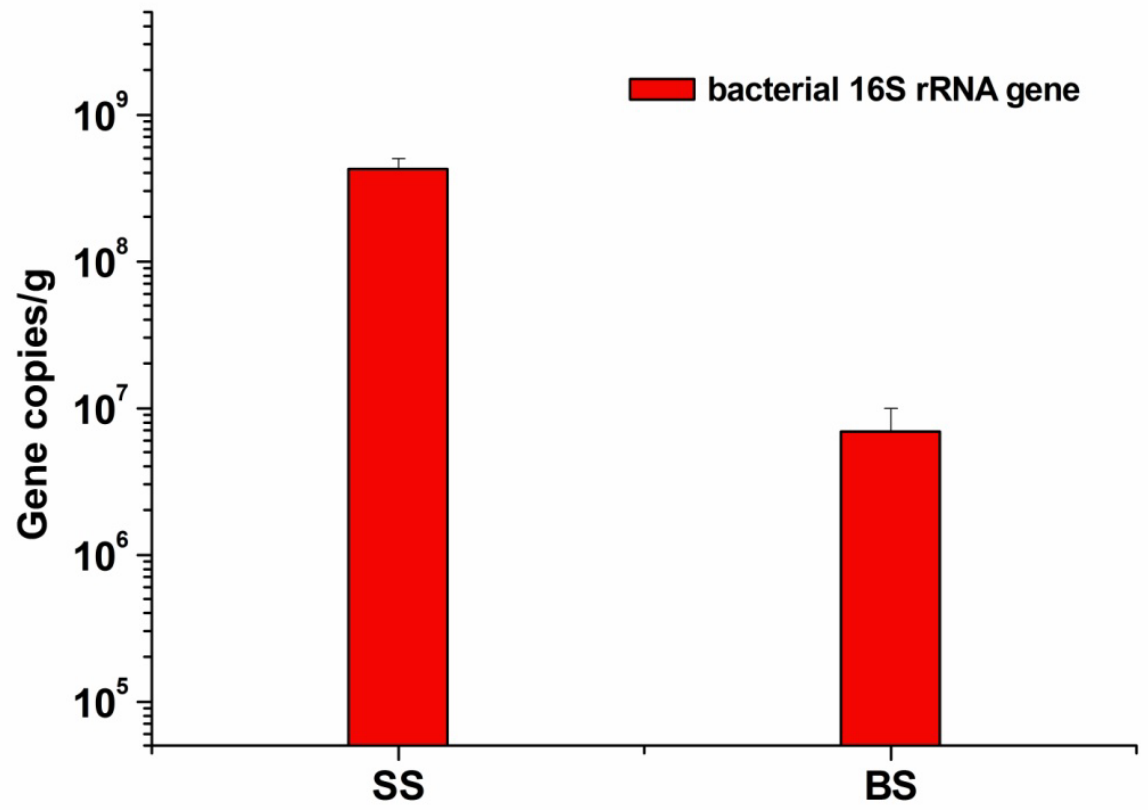

Figure S1. Abundance of bacterial 16S rRNA genes for SS and BS samples collected from Gongxiaoshe hot spring. Data are expressed as gene copies per gram of sediment (dry weight). Error bars represent the standard deviation of the mean $(n=3)$. 


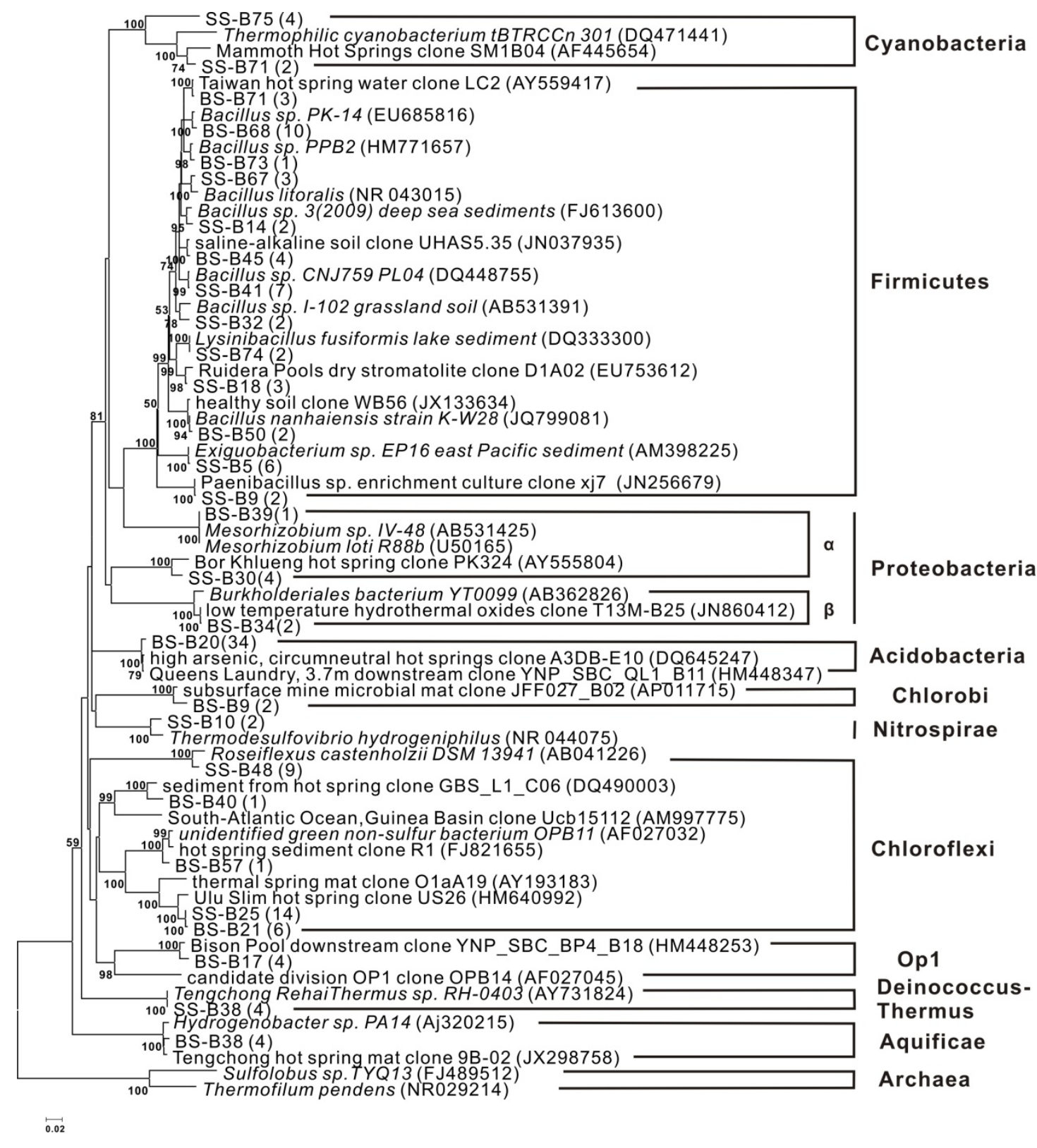

Figure S2. The bacterial 16S rRNA phylogenetic tree is constructed using neighbor-joining analysis from the sediments of the Gongxiaoshe hot spring (SS and BS). Bootstrap confidence values are obtained using 1000 replicates, and values greater than $50 \%$ are indicated at the nodes. The scale bar represents the expected number of changes per nucleotide position. 


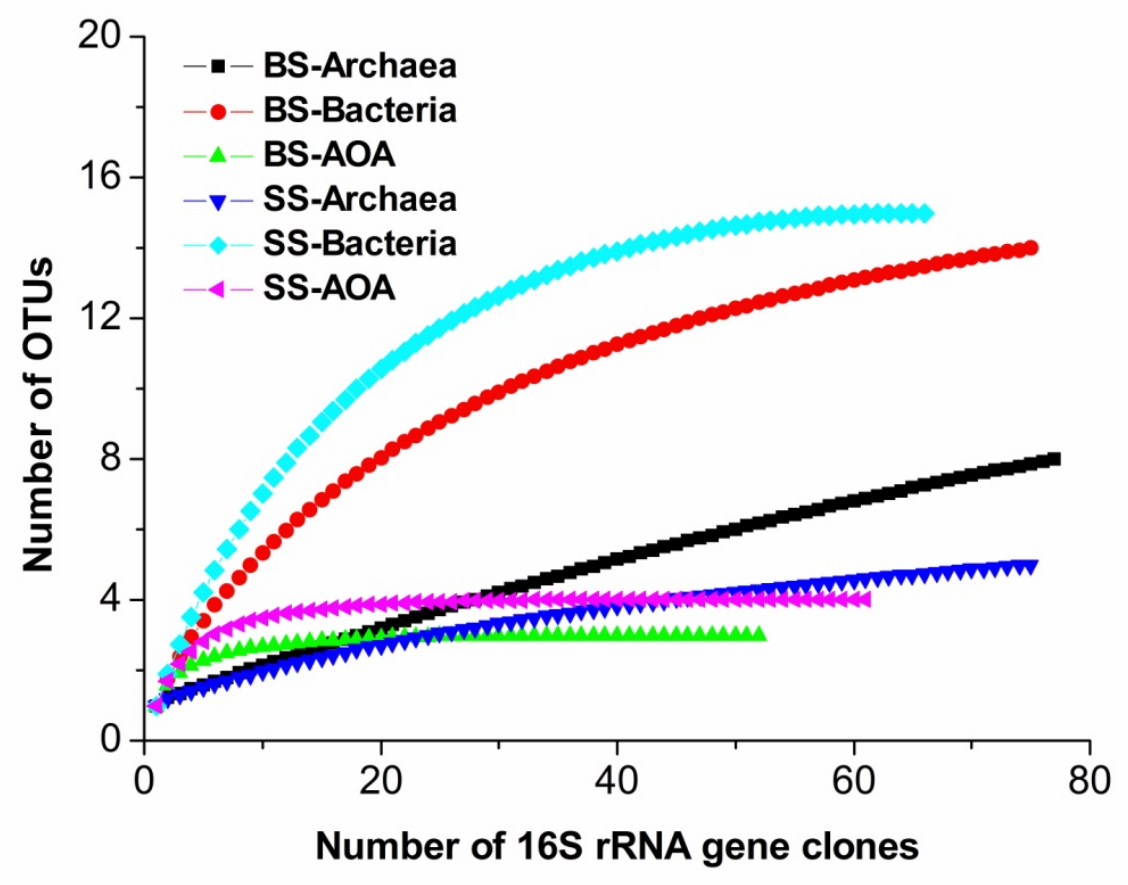

Figure S3. Rarefaction curve of the 16S rRNA gene and functional gene (AOA) clone libraries constructed for SS and BS samples from the Gongxiaoshe hot spring. 